\title{
On Gaussian Random Neuronal Field Model: Moment Neuronal Network Approach
}

\author{
Wenlian Lu, Member, IEEE
}

Jianfeng Feng

\begin{abstract}
A novel model is proposed to describe the rich dynamics of spiking activities of leaky integrate-and-fire (LIF) neuronal networks via the moment neuronal network approach. Different from the existing neuronal field model (for example, Wilson-Cowan-Amari model) which only takes the first-order moment (mean firing rate) into considerations, we develop a Gaussian random field to qualitatively describe the spatiotemporal distribution of the first- and second-order moments: mean firing rate, variance or coefficient of variation (CV) equivalently, and the coefficient of correlation (CC), of spiking trains. By this neuronal field model, we find out that the firing rate response with respect to the input may be not sigmoidal or even monotonic if the inhibition is stronger than excitation, which leads fruitful dynamical behaviors, in comparison with the sigmoidal response. In addition, within this framework, we can analyse the synchronisation propagation in the LIF neuronal network. We use our Gaussian random field model to investigate how the three key factors: the ratio between inhibition and excitation, the size of synchronous cluster, and the background firing rate, decide the stability of a synfire chain.
\end{abstract}

\section{INTRODUCTION}

Recent years have witnessed a significant growth in the field of biological computation [1], [2], [3], which is bridging the gap between the experimental data and theoretical neuroscience. Computational neuronal models, say, the LIF model [4] and the more biophysical Hodgkin-Huxley model [5], provide us with a more powerful tool to analyzing the dynamics of actual biological neurons than the traditional classical approach to neural modelling. The spike activities, which are usually described as point processes, are considered as the main functional medium to carry the neural signal, regarded as a function of the membrane potential and concentration of ions.

When dealing with a neuronal network, these existing models exhibit complicated dynamical behaviours and seems impossible to be analytically treated in a rigorous mathematical manner. Furthermore, there exists a gap between the computational neuronal model and employing stochastic methods. On the other hand, the general stochastic model, for example, general linear model (GLM), has a good performances in neural signal processing but the model is too simple to be linked with the underlying biological background.

Wenlian Lu is jointly with the Laboratory for Mathematical Neuroscience RIKEN Brain Science Institute Hirosawa 2-1, Wako-shi, Saitama 3510198, JAPAN, and the Centre for Computational Systems Biology, Fudan University, Shanghai, P. R. China( email: wenlian@ fudan.edu.cn).

Jianfeng Feng is with the Centre for Scientific Computing, Warwick University, Coventry CV4 7AL, UK, and the Centre for Computational Systems Biology, Fudan University, Shanghai, P. R. China (email: jianfeng.feng@warwick.ac.uk).
A possible approach to bridge this gap is to develop a model based on the biological description of neuronal networks with proper simplifications and approximations so that some well-developed dynamical theory and statistic methods can be employed to associate model activities with experimental data.

So, it is not surprising that attempting to describe neuronal network spike activities with partial differential equations (PDE) or field equations (FE) has been taken up by many authors and is still an active topic [13]. A classical approach is the well-known Wilson-Cowan-Amari (WCA) model [6], [7], [8], [9], which describes the firing rates of neural populations by integro-differential equations. In this model, the membrane potential is directly mapped to the firing rate, via the sigmoidal function. The model is widely applied to simulate or mimic the local field potentials (LFP), functional magnetic resonance imaging (fMRI), electroencephalogram (EEG), and magnetoencephalogram (MEG) [10]. However, the properties of neuronal spikes can only be described by their firing rates and, despite succeeding many applications, can not exactly match some aspects to some key biological meaningful quantities, as detailed below.

In this paper, we establish a spatio-temporal Gaussian neuronal field to describe the dynamics of spike activities. The basic approach is the mathematical framework called moment neuronal networks (MNN) [11], which describes the maps of the first and second order (or even higher) statistics from the input spike trains to the output. Also, taking the coefficients of correlation (CC) into considerations [12], we construct evolution equations of the first- and second-order moment statistics: mean firing rate, coefficient of variation (or equivalently variance), and CC. In contrast to all approaches in the literature such as the mean field approach (WCA model), our approach is, essentially, to describe the neuronal activity as a Gaussian random field [14]).

By including higher-order moment statistics, our approach can describe more aspects of the properties in spiking network activities. As one would expect, when the variance in our model is removed, the firing rate response with respect to the input becomes sigmoidal, which implies that in this situation our continuous-time model has the same property as the WCA model. Instead, if the inhibition is stronger than the excitation, in a reasonable parameter area, the map of the firing rate is non-sigmoidal and non-monotonic, similar to the tent or logistic map. Synchronisation, a wellknown biological phenomenon, can be well described as the correlation between spike trains, one of the second order statistics [12], [15], [16]. It has been earlier reported in the 
literature that a feed-forward spiking network can easily lead to the propagation of a synfire chain [17], [18]. The MNN framework allows us to theoretically analyse it, under some mild conditions.

\section{MODEL DESCRIPTION}

Our main approach is the theory of moment neuronal network [11]. The main idea is to represent (approximate) the spike activity of a neuron (a point process) by a continuous process in the spirit of the ergodicity and central limit theorem. In details, we start with a LIF neuronal network with $p$ neurons and model the potential activity of the $i$-th neuron as follows:

$d V_{i}(t)=-L V_{i}(t) d t+\sum_{j} w_{i j}^{E} d N_{j}^{i, E}(t)+\sum_{j} w_{i j}^{I} d N_{j}^{i, I}(t)$,

where $1 / L$ is the leaky resistance, $w_{i j}^{E}, w_{i j}^{I}$ is the EPSP, IPSP size from the $j$-th pre-synaptic neuron $(j=1,2, \cdots, p)$, and $N_{j}^{i, E}(t)$ and $N_{j}^{i, I}(t)$ are random point processes of the synaptic spike stimulus. First, we regard the spike point process as the renewal processes:

$$
d N_{j}^{i, v}(t)=\sum_{k} \delta\left(t-\sum_{l=1}^{k} T_{j, l}^{i, v}\right) d t
$$

with the $l$-th inter-spike interval (ISI) $T_{j, l}^{i, v}$ with $v=E$ or $I$ supposed to be independently identically distributed. Since the number of renewals converge to a Gaussian random variable due to the central limit theorem, second, we have the following approximation as $t$ is large:

$$
d N_{j}^{i, v}(t) \sim \mu_{j}^{i, v} d t+\sigma_{j}^{i, v} d B_{j}^{i, v}
$$

where $B_{j}^{i, v}, j=1, \cdots, p$ are correlated Brownian motions. And, according to the renewal theorem [19],

$$
\mu_{j}^{i, v}=\frac{1}{T_{\text {ref }}+\left\langle T_{j, 1}^{i, v}\right\rangle}, \quad\left(\sigma_{j}^{i, v}\right)^{2}=\frac{\operatorname{var}\left(T_{j, 1}^{i, v}\right)}{\left\langle T_{\text {ref }}+T_{j, 1}^{i, v}\right\rangle^{3}},
$$

where $T_{\text {ref }}$ represents the refractory period and $\langle\cdot\rangle$ and $\operatorname{var}(\cdot)$ denotes the expectation and variance of a random variable, respectively.

Thus, Eq. (1) yields as Ornstein-Uhlenbeck (OU) processes:

$$
d V_{i}(t)=-L V_{i}(t) d t+\bar{\mu}_{i} d t+\bar{\sigma}_{i} d B
$$

with a standard Brownian motion (Wiener process) $B_{t}$,

$$
\begin{aligned}
\bar{\mu} & =\sum_{j}\left[w_{i j}^{E} \mu_{j}^{i, E}+w_{i j}^{I} \mu_{j}^{i, v}\right], \\
\bar{\sigma} & =\sqrt{\sum_{j} \sum_{k} \sum_{v, u=I, E} \sigma_{j}^{i, v} \sigma_{k}^{i, u} \rho_{j, k}^{i, v, u}},
\end{aligned}
$$

where $\rho_{j, k}^{i, v, u}$ is the correlation coefficient between $d N_{j}^{i, v}$ and $d N_{k}^{i, u}$. Initiated with a rest potential $V_{r}$, when the potential of the $i$-th neuron reaches a threshold $V_{t h}$, the neuron emits a spike at that time and is rest back to $V_{r}$ after a refractory period $T_{\text {ref }}$.
In the term of Siegert's expression [3], we can have the expression of all moments of the output stationary inter-spike interval (ISI) distribution based on the Fokker-Planck equation and first-passage-time (FPT) theory [28]. In particular, the mean and the variance of the output ISI are

$$
\begin{aligned}
\left\langle T_{\text {out }, i}\right\rangle & =\frac{2}{L} \int_{I\left(V_{r}, \bar{\mu}_{i}, \bar{\sigma}_{i}\right)}^{I\left(V_{t h}, \bar{\mu}_{i}, \bar{\sigma}_{i}\right)} D_{-}(u) d u \\
\operatorname{Var}\left(T_{\text {out }, i}\right) & =\frac{8}{L^{2}} \int_{I\left(V_{r}, \bar{\mu}_{i}, \bar{\sigma}_{i}\right)}^{I\left(V_{t h}, \bar{\mu}_{i}, \bar{\sigma}_{i}\right)} D_{-} \otimes D_{-}(u) d u .
\end{aligned}
$$

Here,

$$
\begin{aligned}
I(\xi, y, z) & =\frac{\xi L-y}{z} \\
D_{-}(u) & =\exp \left(u^{2}\right) \int_{-\infty}^{u} \exp \left(-v^{2}\right) d v \\
D_{-} \otimes D_{-}(u) & =\exp \left(u^{2}\right) \int_{-\infty}^{u} \exp \left(-v^{2}\right) D_{-}^{2}(v) d v
\end{aligned}
$$

where $D_{-}(u)$ is exactly the Dawson's integral. We continue approximating the output spike trains as renewal processes. Using the renewal theory, we can obtain the mean and variance of the output spiking as

$$
\begin{aligned}
\mu_{\text {out }, i} & =\frac{1}{T_{r e f}+\frac{2}{L} \int_{I\left(V_{r}, \bar{\mu}_{i}, \bar{\sigma}_{i}\right)}^{I\left(V_{t h}, \bar{\sigma}_{i}\right)} D_{-}(u) d u}, \\
\sigma_{\text {out }, i} & =\frac{\left(\frac{8}{L^{2}} \int_{I\left(V_{r}, \bar{\mu}_{i}, \bar{\sigma}_{i}\right)}^{I\left(V_{t h}, \bar{\sigma}_{i}, \bar{\sigma}_{i}\right)} D_{-} \otimes D_{-}(u) d u\right)^{1 / 2}}{\left(T_{\text {ref }}+\frac{2}{L} \int_{I\left(V_{r}, \bar{\mu}_{i}, \bar{\sigma}_{i}\right)}^{I\left(V_{t}, \bar{\sigma}_{i}, \bar{\sigma}_{i}\right)} D_{-}(u) d u\right)^{3 / 2}} .
\end{aligned}
$$

For simplicity of depiction in the following, we define

$$
\begin{aligned}
& S_{1}(y, z) \doteq \frac{1}{T_{r e f}+\frac{2}{L} \int_{I\left(V_{r}, y, z\right)}^{I\left(V_{t h}, y, z\right)} D_{-}(u) d u}, \\
& S_{2}(y, z) \doteq \frac{\left(\frac{8}{L^{2}} \int_{I\left(V_{r}, y, z\right)}^{I\left(V_{t h}, y, z\right)} D_{-} \otimes D_{-}(u) d u\right)^{1 / 2}}{\left(T_{r e f}+\frac{2}{L} \int_{I\left(V_{r}, y, z\right)}^{I\left(V_{t h}, y, z\right)} D_{-}(u) d u\right)} .
\end{aligned}
$$

In addition, to study the correlation evolving equations, we firstly consider two OU processes driven by two correlated Brownian motions, as shown in [12], we can regard the CC of the output spike trains as a function of the $\mathrm{CC}$ of the input Brownian motions:

$$
\rho_{\text {out }}=\Phi\left(\rho_{\text {in }}\right) \text {, }
$$

The map of cc has been studied in details by [12], [11]. By numerical illustrations and direct reasoning, they both found that $\Phi(\cdot)$ satisfies: (1). $\Phi(0)=0, \Phi_{1}=1 ;(2) . \Phi(\cdot)$ is increasing and below the identical map in $[0,1]$, i.e., $\Phi(\rho) \leq$ $\rho$ for all $\rho \in[0,1]$; (3). $\Phi(\cdot)$ depends on the input firing rate and $\mathrm{CV}$ (variance) and trends to the identical map in $[0,1]$ if the mean firing rates in the period of the time-scale $1 / L$ is large via increasing the input firing rate or CV. By the linear perturbation theory [20], we can derive the slopes of the map $\Phi(\cdot)$ near $\rho_{\text {in }}=0$ and $\rho=1$ respectively. 

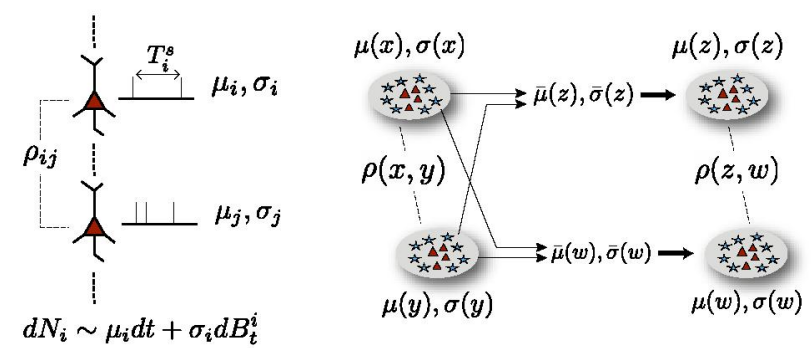

Fig. 1. Approximation described by the map $\Gamma$ : (A): the Gaussian approximation of the pointer process; (B): the map of $\Gamma$ in a spiking neuronal network.

Thus, if the map of CC is well defined, we are in the position to derive a map $\Gamma$, with respect to the mean, variance (CV), and CC, from the input spiking trains to the output in the neuronal network as:

$$
\begin{array}{ll}
\Gamma:\left(\mu_{\text {in }}, \sigma_{\text {in }}, \Sigma_{\text {in }}\right) & \rightarrow\left(\mu_{\text {out }}, \sigma_{\text {out }}, \Sigma_{\text {out }}\right) \\
\mu_{\text {out }, .} & =S_{1}(\bar{\mu} ., \bar{\sigma} .) \\
\sigma_{\text {out },} & =S_{2}\left(\bar{\mu}_{.}, \bar{\sigma} .\right) \sqrt{S_{1}\left(\bar{\mu}_{.}, \bar{\sigma} .\right)} \\
\rho_{\text {out }, i, j} & =\Phi_{i, j}\left(\rho_{\text {in }, i, j}\right),
\end{array}
$$

where $\rho_{\text {input }}(i, j)$, the correlation of the input spiking trains of the $i$-th and $j$-th neurons, has the form:

$$
\rho_{i n}(i, j)=\frac{\left(w_{i} . \sigma_{.}\right)^{\top} \Sigma\left(w_{j} . \sigma_{.}\right)}{\sqrt{\left(w_{i} . \sigma_{.}\right)^{\top} \Sigma\left(w_{i} . \sigma_{.}\right)} \sqrt{\left(w_{j} . \sigma .\right)^{\top} \Sigma\left(w_{j} . \sigma .\right)}} .
$$

Equivalently, sometimes in this paper, we can consider the coefficient of variation (CV) of the ISI, instead of the variance $\sigma$ :

$$
C V_{i}=\frac{\sqrt{\operatorname{Var}\left(T_{i}\right)}}{\left\langle T_{i}\right\rangle}=\sqrt{\frac{\sigma_{i}^{2}}{\mu_{i}}} .
$$

Thus, we have

$$
C V_{\text {out }, \cdot}=S_{2}\left(\bar{\mu}_{.}, \bar{\sigma}_{.}\right)
$$

The moment neuronal network map can be described by three variables, $(\mu, \sigma, \rho)$, as depicted in Fig. $1 \mathbf{A}$ and $\mathbf{B}$. Using this Gaussian motion as the input for the next iteration, we can model the neuronal activities as a Gaussian field. This is the main idea of this paper. We regard again the output of a LIF network model as a renewal process and Gaussian motions. So, these three classes of variables (firing rate, variance, $\mathrm{CV}$, and $\mathrm{CC}$ ) can fully describe the output Gaussian motions. Then, we derive evolution equations over these three classes of variables using the moment map. Thus, the spiking neuronal network is described by a Gaussian field.

Now, we develop a Gaussian field of discrete time to model the underlying neuronal networks. First, we consider a feedforward neuronal field by iterating the maps of moments to derive difference equations. Let $\mu(x, k)$ and $\sigma(x, k)$ the mean and variance at the location $x$ of the $k$-th layer and $\rho(x, y, k)$ the correlation between the location $x$ and $y$ at the $k$-th layer. Let $w(x, y)$ be the interconnection coefficient between the locations $x$ and $y$. Define

$$
\begin{aligned}
& w * \mu(x, k)=\int w(x, y) \mu(x, k) d y \\
& \langle w * \sigma, w * \sigma\rangle_{\rho(k)}(x, y, k) \\
& =\int w(x, u) w(y, v) \sigma(u, k) \sigma(v, k) \rho(u, v, k) d u d v .
\end{aligned}
$$

Then, $\|w * \rho\|_{\rho(t)}^{2}(x, k)=\langle w * \sigma, w * \sigma\rangle_{\rho(t)}(x, x, k)$. Thus, the neuronal field yields the following iterative equations:

$$
\left\{\begin{array}{l}
\mu(x, k+1)=S_{1}\left(w * \mu(x, k),\|w * \sigma\|_{\rho(k)}(x, k)\right) \\
\sigma(x, k+1)=S_{2}\left(w * \mu(x, k),\|w * \sigma\|_{\rho(k)}(x, k)\right) \\
\sqrt{S_{1}\left(w * \mu(x, k),\|w * \sigma\|_{\rho(k)}(x, k)\right)} \\
\rho(x, y, k+1)=\Phi_{i, j, k}\left[\frac{\langle w * \sigma, w * \sigma)_{\rho(k)}(x, y, k)}{\|w * \sigma\|_{\rho(k)}(x, k)\|w * \sigma\|_{\rho(k)}(y, k)}\right]
\end{array}\right.
$$

where $k=1,2, \cdots$, . Sometime, we consider the iterative equations of $C V(x, k)$, instead of $\sigma$, as follows:

$$
C V(x, k+1)=S_{2}\left(w * \mu(x, k),\|w * \sigma\|_{\rho(k)}(x, k)\right) .
$$

In the continuous-time case, we can derive differential equations to describe their evolution directly from the map iterations. We use a slow time scale such that the spike processes keep stationary. Let $t$ be continuous time which is much slower than that of the sampling. Thus, we can use the moment map to describe the dynamics of the moments of spike activities. Letting $\tau$ be the sampling time interval, the iterative equations (6) yields the following integrodifferential equations:

$$
\left\{\begin{aligned}
\tau \frac{\partial \mu}{\partial t}= & -\mu+S_{1}\left(\omega * \mu,\|\omega * \sigma\|_{\rho}\right) \\
\tau \frac{\partial \sigma}{\partial t}= & -\sigma+S_{2}\left(\omega * \mu,\|\omega * \sigma\|_{\rho}\right) . \\
& \sqrt{S_{1}\left(\omega * \mu,\|\omega * \sigma\|_{\rho}\right)} \\
\tau \frac{\partial \rho}{\partial t}= & -\rho+\Phi_{x, y, t}\left(\frac{\langle\omega * \sigma, \omega * \sigma\rangle_{\rho}}{\|\omega * \sigma\|_{\rho}(x)\|\omega * \sigma\|_{\rho}(y)}\right)
\end{aligned}\right.
$$

where $t \in R$, in addition with its $\mathrm{CV}$ :

$$
\tau \frac{\partial C V}{\partial t}=-C V+S_{2}\left(\omega * \mu,\|\omega * \sigma\|_{\rho}\right) .
$$

These maps can well approximate the dynamics of LIF neuron model. A lots of numerical illustrations are conducted in the literature [11], [12]. In the current paper, we present two examples of the dynamics analysis of the evolution equations we derived above.

\section{NON-SIGMOID FIRING RATE RESPONSE}

Consider the situation with the input variance trending to zero and $r<1$, it can be proved in [21] that the sub-manifold with $\sigma=0$ is stable and the system converges to

$$
\left\{\begin{aligned}
\tau \cdot \frac{\partial \mu}{\partial t} & =-\mu+\mathcal{L}(\omega * \mu) \\
\sigma & =0,
\end{aligned}\right.
$$

where the firing rate response function $\mathcal{L}(\cdot)$ is a sigmoid-type function:

$$
\mathcal{L}(\nu)= \begin{cases}\frac{1}{T_{r e f}+\frac{2}{L} \ln \sqrt{\frac{(1-r) \nu-V_{r} L}{(1-r) \nu-V_{t h} L}}} & \nu>V_{t h} L /(1-r) \\ 0 & 0<\nu<V_{t h} L /(1-r) .\end{cases}
$$


However, if $r>1$, i.e, the inhibition is stronger than excitation, it is not always the case. Since the maps of moments are rather complicated, here, for simplicity, we assume that

$$
\begin{aligned}
& w_{i j}^{I}=-r w_{i j}^{E}, \quad \Sigma=\{\rho(i, j)\}=\Sigma^{E}=\Sigma^{I}, \\
& \mu_{j}=\mu_{j, 1}^{i, E}=\mu_{j, 1}^{i, I}, \sigma_{j}=\sigma_{j, 1}^{i, E}=\sigma_{j, 1}^{i, I},
\end{aligned}
$$

where $r$ indicates the proportion between inhibitory and excitatory input. $r=1$ means that the input is exactly balanced, but $r>1$ means that inhibitory input is stronger than excitatory input and the vise versa. Further simplification is conducted by unifying the neurons. That is, $\mu_{i}, \sigma_{i}$, and $\rho_{i j}$ are regarded invariant with respect to $i$. So, we use a single spiking neuron representing a homogeneous neuronal network. We define the mean and the variance of the input from $p$ excitation neurons and $p$ inhibition neurons:

$$
\begin{aligned}
\bar{\mu} & =p(1-r) \mu_{i n}, \\
\bar{\sigma} & =\sqrt{\left(1+r^{2}\right)(p+p(p-1) \rho)} \sigma_{i n},
\end{aligned}
$$

which are obtained from the assumption that the excitation synaptic spike train and inhibition synaptic spike train are independent; in the same group, the CC between two excitation (or inhibition) synaptic inputs is commonly $\rho$; the variance of each synaptic spike input is commonly $\sigma_{i n}$.

Recall the firing rate response function in the homogeneous MNN map $\Gamma$ :

$$
S_{1}(\mu, \sigma)=\frac{1}{T_{r e f}+\frac{2}{L} \int_{I\left(V_{r}, \mu, \sigma\right)}^{I\left(V_{t h}, \mu, \sigma\right)} D_{-}(u) d u} .
$$

With a fixed $C V>0$ and a zero $\mathrm{CC}$, its monotonicity is inverse to that of the following function

$$
f(\mu)=\int_{\frac{V_{r} L-(1-r) \mu}{\sqrt{\left(1+r^{2}\right) p} \sigma}}^{\frac{V_{t h} L-p(1-r) \mu}{\sqrt{\left(1+r^{2}\right) p}}} \exp \left(u^{2}\right) d u \int_{-\infty}^{u} \exp \left(-v^{2}\right) d v .
$$

Thus, owing to $\sigma=C V \sqrt{\mu}$, we obtain the derivative of $f$ as follows:

$$
\begin{aligned}
\frac{\partial f}{\partial \mu}= & \frac{\left[-p(1-r) \mu-V_{t h} L\right]}{2 \sqrt{\left(1+r^{2}\right) p} C V \mu^{3 / 2}} \exp \left(I_{+}^{2}\right) \int_{-\infty}^{I_{+}} \exp \left(-v^{2}\right) d v \\
& +\frac{(r-1) p+V_{r} L}{2 \sqrt{\left(1+r^{2}\right) p \mu} C V} \exp \left(I_{-}^{2}\right) \int_{-\infty}^{I_{-}} \exp \left(-v^{2}\right) d v
\end{aligned}
$$

where

$$
I_{+}=\frac{V_{t h} L-p(1-r) \mu}{\sqrt{\left(1+r^{2}\right) p} \sigma}, I_{-}=\frac{V_{r} L-(1-r) \mu}{\sqrt{\left(1+r^{2}\right) p} \sigma},
$$

according to $V r=0$ as we set.

We consider two situations: a small $\mu$ near zero and a large $\mu$ near infinity. On one hand, in the case of $\mu \rightarrow 0+$, we have

$$
\frac{\partial f}{\partial \mu} \sim \frac{-V_{t h} L}{2 \sqrt{\left(1+r^{2}\right) p} C V \mu^{3 / 2}} \exp \left(I_{+}^{2}\right) \int_{-\infty}^{I_{+}} \exp \left(-v^{2}\right) d v
$$

by neglecting the terms of higher orders. One can see that whether $r>1$ or not, $f$ is monotonically increasing with respect to small $\mu$, which implies that the firing rate response increases with respect to the ascent of a small input firing rate.

On the other hand,in the case of $\mu \rightarrow+\infty$, we have

$$
\begin{aligned}
& \frac{\partial f}{\partial \mu} \sim \frac{[p(r-1)]}{2 \sqrt{\left(1+r^{2}\right) p \mu} C V}\left[\exp \left(I_{+}^{2}\right) \int_{-\infty}^{I_{+}} \exp \left(-v^{2}\right) d v\right. \\
& \left.+\exp \left(I_{-}^{2}\right) \int_{-\infty}^{I_{-}} \exp \left(-v^{2}\right) d v\right],
\end{aligned}
$$

by neglecting the terms of lower orders. It is clear that if $r<1$, the firing rate response still increases according an ascent and large input firing rate; but if $r>1$, with a stronger inhibition than excitation, the situation turns over that the firing rate response decreases with respect the input firing rate of relative large values.

In Fig. (2), we plot the surfaces of the firing rate response with respect to $\left(\mu_{i n}, C V_{i n}\right)$ for the cases of $r<1$ and $r>$ 1. As concentrating on the inside, we also plot the curves of the firing rate response with respect to the input firing rate or $\mathrm{CV}$ respectively, also as shown in Fig. 2. One can see that the firing rate response is monotonically increasing with respect to the input firing rate and has a sigmoid-like curve in the case of $r<1$. Instead, if $r>1$, the firing rate response is not sigmoid or monotonic any more. As the bottom-right panel in Fig. 2 shows, it bends like a tent map or logistic map if the inhibition is strong. Since it is well known that this shape of map in an iteration equation may lead to complicated dynamical behaviours such as bifurcations even chaos, it is reasonable to expect the dynamics exhibits such rich behaviours. This will be one of our future research objectives to establish a clear spectrum of this neuronal field dynamics.

We also give a simple illustration of the attractor diagram of $(\mu, C V)$ with respect to $r$. As Fig. (3) shows, the system can keep a point attractor with positive firing rate, which may correspond to the well-known spontaneous activity in realworld neuronal networks [22], [23]. In details, for $r<0.8$ or so, the pair $(\mu, C V)$ has deterministic point attractor that $\mu$ converges to some positive value but $C V$ to zero. That is, for $r<0.8$, the system trend to a deterministic system with $\sigma=0$, coinciding with what we show in the beginning of this section. Instead, for a large $r$, even a single neuron (or a homogeneous neuronal network) can have more fruitful dynamical behaviours. First, for $r \in(0.8,1)$, the system has a sub-Poisson point attractor with a positive firing rate and nonzero $\mathrm{CV}$ less than 1. For $r \in(1,3.25)$, the region between two red lines, the system becomes multi-stable with two point attractor: one is a quasi-silent Poisson attractor with almost zero firing rate and $C V=1$ and the other is with positive firing rate and a positive $\mathrm{CV}$ from sub-Poisson to sup-Poisson. As we show, in the region of biological reasonable parameters, we obtain a fixed point attractor of a low but positive firing rate (less than $20 \mathrm{HZ}$ ) spontaneous activity, accompanied with the situation that the inhibitory input should be stronger than the excitatory input.

Fig. 4 shows the bifurcation diagram of $(\mu, C V)$ with 

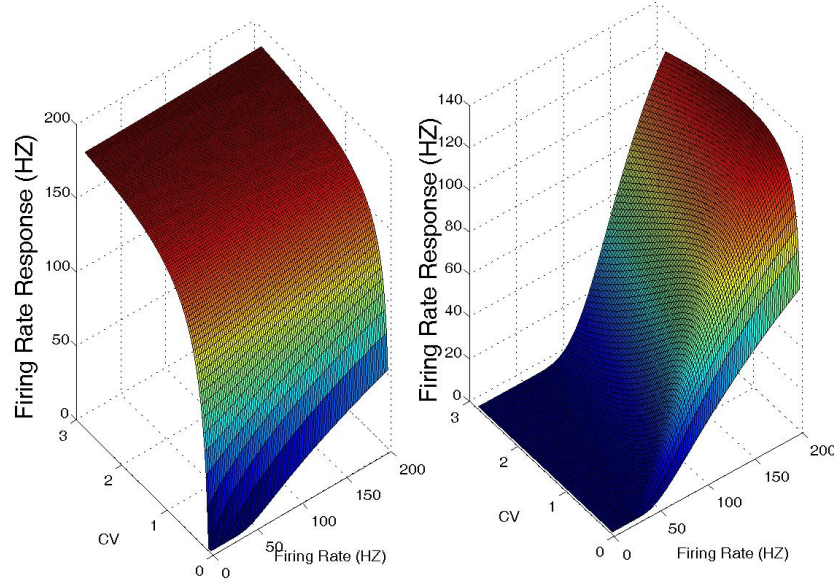

Fig. 2. Maps of the firing response with respect to the firing rates and $\mathrm{CV}$ of the input. Here, we pick the size of network as $1 / L=20$ (msec), $p=100, V_{t h}=0(\mathrm{mv}), V_{r}=-20(\mathrm{mv})$, and $T_{r e f}=5(\mathrm{msec})$. The two surface plots at the top illustrates the map of the firing rate response with respect to $\left(\mu_{i n}, C V_{i n}\right)$ in the formula (9) for $r=0.5$ (left) and $r=1.5$ (right). The four plots below are the curves of the firing rate response with respect to $\mu_{i n}$ or $C V_{i n}$ with the other fixed. They are the firing rate response with respect to $\mu_{i n}$ with $C V=0.6$ and $r=0.5$ (middle-left), $C V=1.5$ and $r=1.5$ (bottom-left), the firing rate response with respect to $C V_{i n}$ with $\mu=10$ (HZ) and $r=0.5$ (middle-right), $\mu=10$ (HZ) and $r=1.5$ (bottom-right).
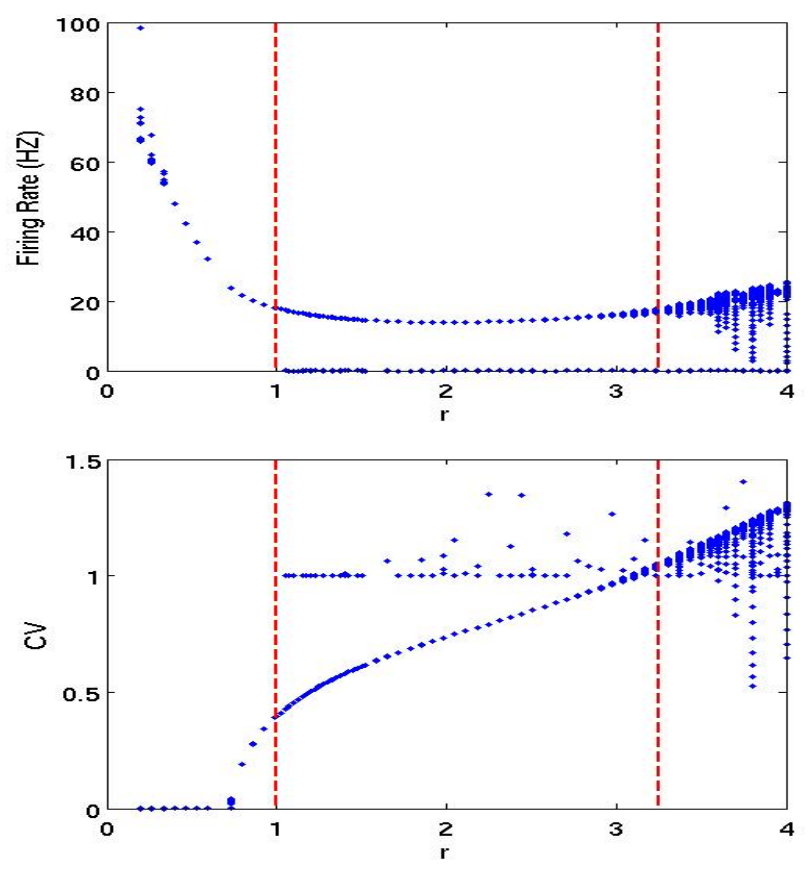

Fig. 3. Stability diagram of the map $\Gamma$ with respect to the parameter $r$ with a fixed $\rho=0$. The parameters are picked as the same as in Fig. 2. We plot the collection of the states of 50 iterations after initial 100 iterations of the map $S_{1,2}(\cdot)$ with 30 overlaps of randomly selected initial data. The regions between red broken lines in both figures corresponds to the spontaneous activities.
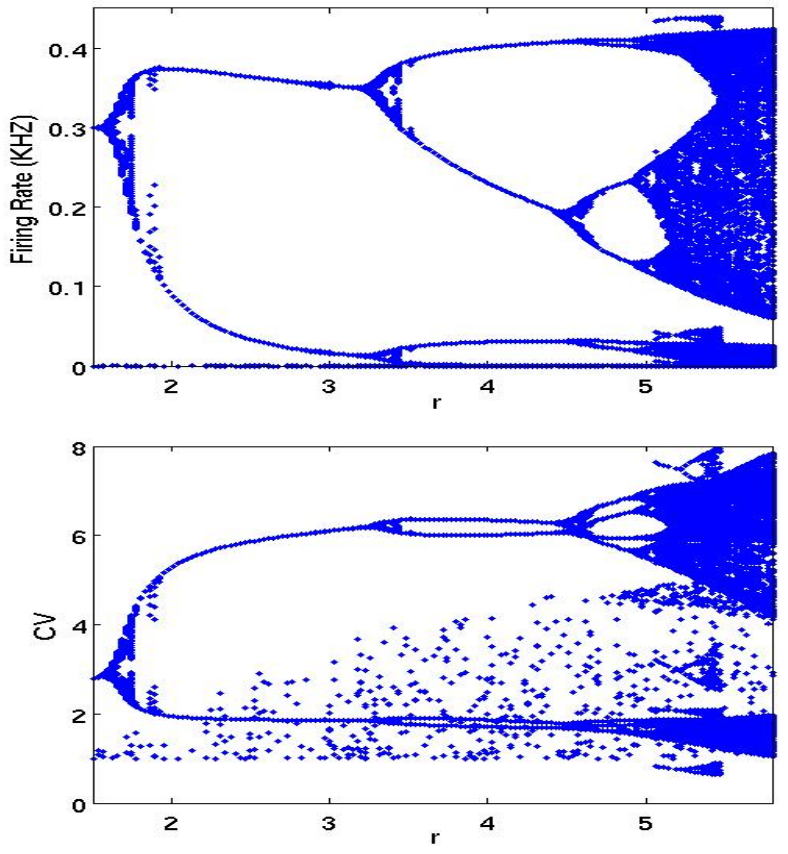

Fig. 4. Bifurcation diagram of the map $\Gamma$ with respect to the parameter $r$ with a fixed $\rho=0$. The parameters are picked as $1 / L=20$ (msec), $p=1000, V_{t h}=0(\mathrm{mv}), V_{r}=-20(\mathrm{mv})$, and $T_{\text {ref }}=2(\mathrm{msec})$. We plot the collection of the states of 50 iterations after initial 100 iterations of the map $S_{1,2}(\cdot)$ with 30 overlaps of randomly selected initial data.

respect to $r$. With selective parameters, one can see that the dynamics have a periodic-doubling phenomenon and trend a chaotic-like attractor as $r$ increases. In Fig. 5, we plot the phase of the firing rate and $\mathrm{CV}$ of an attractor. From numerical calculations, we conclude that its largest Lyapunov exponent equals 0.0864 , which implies that the attractor is chaotic. Moreover, we also calculate its correlation dimension which is around 1.1574. Since it has only one positive Lyapunov exponent, its K-entropy is equal to the largest Lyapunov exponent. We should point out that for most values of parameters, we can observe the periodicdoubling phenomenon but in order to show them clearly, we have chosen a different set of values of parameter from the previous setting which is obviously biologically more realistic. Certainly the nonlinear dynamics will be further investigated somewhere else in our future work.

\section{SYNCHRONISATION PROPAGATION}

Synfire chain has been widely studied in both experiment and theoretical manners. It is a well-known fact in the literature that in a feed-forward spiking neuronal network, spikes of different neurons could easily synchronize and propagate their activity [18], [24]. This phenomenon can not be described by the WCA model, since it can only concern the first moment of the spiking activity but synchronisation should be described by a second-order statistics. In [25], [26], the authors proposed a computational approach, called "pulse packets", to analyse it via the timing information of spikes. However, it is more direct and clear to describe synchrony 


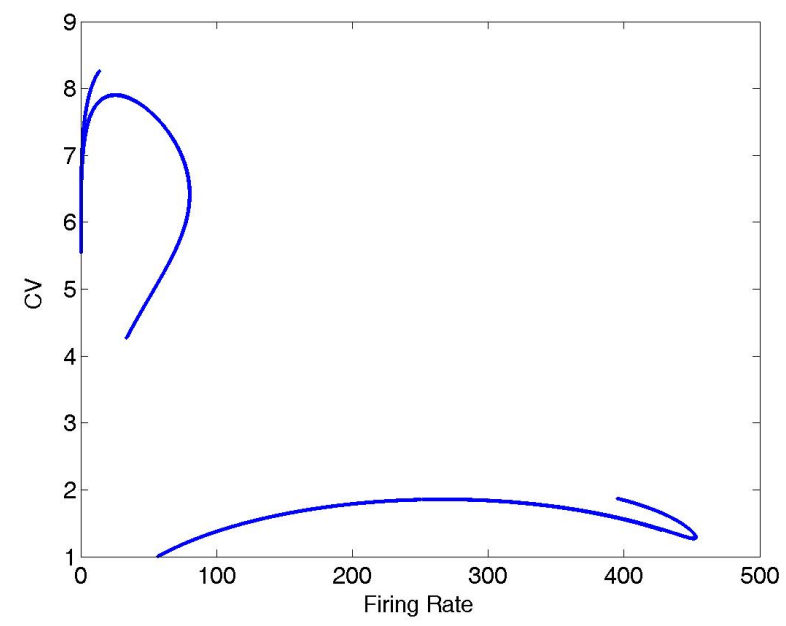

Fig. 5. Firing rate vs. CV phase plot of the attractor of the map $\Gamma$ with with a fixed $\rho=0$. The parameters are picked as $1 / L=20(\mathrm{msec}), p=1000$, $V_{t h}=0(\mathrm{mv}), V_{r}=-20(\mathrm{mv}), T_{r e f}=2(\mathrm{msec}), r=8.5$. We plot the collection of the states of 2000 iterations after initial 1000 iterations of the map $S_{1,2}(\cdot)$.

between spike trains by their coefficient of correlation (CC). Thus, (complete) synchronisation can be defined as the $\mathrm{CC}$ equal to 1 . In [27], the authors use a simple model to analyse correlation between neurons in a synfire chain. However, they only focused on the synchronisation among the cluster and assume the spike activity background out of the synchronous cluster is stochastically independent, which contradicts with the experimental phenomena and the reasoning. In fact, the spike trains between neurons can not be independent since they has probabilities to received a partial of synchronous spikes. A balanced network (with equal proportion between excitation and inhibition, i.e., $r=1$ ) is a general and common assumption for stable synfire chain.

In this paper, we have established field equations to describe the dynamics of CC in LIF neuronal networks, which suggests a novel approach to study synchrony by a mathematical manner.

In this paper, we consider a multi-layer neuronal network with discrete space. For a pair of neurons $i$ and $j$, the CC between them can be formalized as

$$
\begin{array}{r}
\rho^{k+1}(i, j)=\Phi_{i, j}\left[\frac{\langle\omega * \sigma, \omega * \sigma\rangle_{\rho^{k}}(i, j)}{\|\omega * \sigma\|_{\rho^{k}}(i)\|\omega * \sigma\|_{\rho_{i n}^{k}}(j)}\right], \\
\forall i, j=1, \cdots, p, k=1,2, \cdots .
\end{array}
$$

where $\rho^{k}$ is the CC matrix at the $k$-th layer and $\Phi_{i j}$ is the $\mathrm{CC}$ map between the neuron pair $(i, j)$, depending on their firing rates and $\mathrm{CVs}$.

The stability of a synfire chain is twofold. On the one hand, the $\mathrm{CCs}$ between the cluster are large and near 1 ; on the other hand, the other CCs, excluding among the cluster, are low. First, we study the an extreme case: how a network of the coupled systems (10) converge to 1 . We consider the stability of the equilibrium of $\rho_{i j}=1$ for all $i \neq j$ and present the following results via the linear stability theory.
Proposition 1: Let

$$
\begin{aligned}
& L_{p, q}^{i j}=\Phi_{i j}^{\prime}(1)\left\{\frac{W_{i p} W_{j q}}{\left|\sum_{k} W_{i k}\right|\left|\sum_{l} W_{j l}\right|}\right. \\
& \left.-\frac{1}{2}\left[\frac{W_{i p} W_{i q}}{\left(\sum_{k} W_{i k}\right)^{2}}+\frac{W_{j p} W_{j q}}{\left(\sum_{l} W_{j l}\right)^{2}}\right]\right\}
\end{aligned}
$$

and $L=\left[L_{p, q}^{i j}\right]_{i>j, p>q}$ be an $(n-1) n / 2$-order square matrix. If $\rho(L)<1$, then the equilibrium of $\rho_{i j}=1$ for all $i \neq j$ is asymptotically stable.

As shown in [12], if the firing rate per $1 / L$ is sufficiently high, illustrated as larger than 5 spikes per $1 / L$, then the CC map in the positive region is very close to an identity map, symbolled by $I d$. Then, we have the following result if the CC map is the identity map and the all neurons are excited:

Theorem 1: Suppose $\Phi_{i, j}(\cdot)=I d$ for all $i, j=1, \cdots, p$, all couplings are nonnegative, all self-links are positive, and all variances are greater than some give positive number. Let $\mathcal{G}_{k}$ be the interacting topology from the layer $k$ to $k+1^{1}$. If there exists an positive integer $T$ such that the union graph across any time interval with the length larger than $T$ has spanning trees, then $\lim _{k \rightarrow \infty} \rho_{i j}^{t}=1$ holds for all $i \neq j$.

The proof is omitted here. Theorem 1 indicates that in a high firing background, excitation couplings can synchronize the network (cluster) efficiently, which has been illustrated in experiments [18]. That is, in a synfire chain, the synchrony in a cluster can be conserved through propagation in layers owing to the excitation couplings between the synfire group.

Second, we consider the situation that all CCs keep low values. In a real-world neuronal network, without concentrating external stimulus, the CCs of the whole network keep around $0.1 \sim 0.3$. As shown by the results above, a full exciting network is not good to keep CCs in low values. Thus, it is reasonable to believe that to realize a stable synfire chain, an optimal environment is that in the synchronized group, the neurons are all excited, intra-group graph topology is compact, and the others neurons have proportional excitation and inhibition neurons.

As illustration, we consider a random network of $2 N$ nodes (the set $\mathcal{N}$ ), where $N$ nodes are E-neurons (the set $\mathcal{E}$ ) and the other $N$ are I-neurons (the set $\mathcal{I}$ ). Each node has $K=\lambda N$ E-neuron neighbors and $K$ I-neuron neighbors, which are selected randomly with common probability. Here $\lambda$ is the density of the random network. Let $w_{i j}=1$ if $j \in \mathcal{E}$ and $w_{i j}=-r$ if $j \in \mathcal{I}$, where the parameter $r$ represents the proportion rate between excitation and inhibition. Suppose all variance to be identical. Let $\mathcal{M} \subset \mathcal{E}$ be the neuron cluster where synfire chain is supposed to occur and denote its size as $\# \mathcal{W}=W$. The map of $\mathrm{CC}$ is supposed to be uniform and approximated by:

$$
\Phi(x)=(1-b) x^{n}+b x
$$

\footnotetext{
${ }^{1}$ The interacting topology $\mathcal{G}^{k}$ is defined as a pair of set $\{\mathcal{V}, \mathcal{E}\}$. The node set $\mathcal{V}$ corresponds the neuron set at a single layer, which is supposed to be of the same size for different layers. The edge set $\mathcal{E}$ is defined as follows: If there is a link from the neuron $i$ at the $k$-th layer to the neuron $j$ at the $k+1$-th layer, then it corresponds an edge from $i$ to $j$ in $\mathcal{E}$.
} 

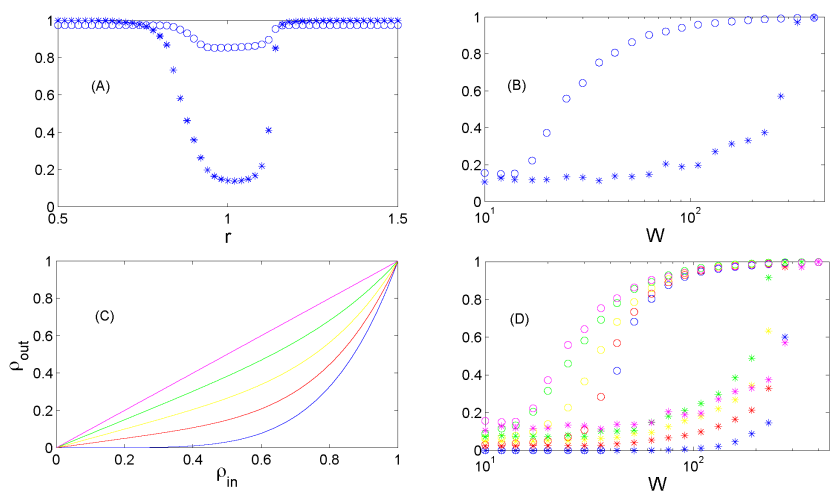

Fig. 6. Coefficients of Correlation attractor of the CC system (10). Here, we pick the size of network as $2000(\mathrm{~N}=1000)$, the density as $\lambda=0.1$, the CC map as $\Phi(\rho)=b \rho+(1-b) \rho^{5}$. (A): the attractors of the mean CC through the cluster $(o)$ and outside $(*)$ with respect to $r$ with a fixed $W=80 ;(\mathbf{B})$ : the attractors of the mean CC through the cluster $(o)$ and outside $*$ with respect to the size of the cluster $W$ with a fixed $r=1 ;(\mathbf{C})$ : the curve plots of the map $\Phi(\cdot)$ with different $b=0$ (blue), 0.25 (red), 0.5 (yellow), 0.75 (green), and 1 (magenta); (D): the attractors of the mean CC through the cluster $(o)$ and outside $(*)$ with respect to the size of the cluster $W$ with a fixed $r=1$, and different $b=0$ (blue), 0.25 (red), 0.5 (yellow), 0.75 (green), 1 (magenta).

where the parameter $b$ is picked in $[0,1]$ and $n$ is an integer larger than 1 . When $b=1, \Phi=I d$. It is a monotonically increasing convex function. Here, we pick $n=5$. We analyse the point attractors $\left(\rho_{i, j}^{*}\right)_{i, j=1}^{p}$ of the system (10) and measure the mean of $\mathrm{CCs}$ among the cluster and the outside:

$C C_{\text {syn }}=\left\langle\rho^{*}(i, j)\right\rangle_{(i, j) \in \mathcal{A}}, C C_{\text {outside }}=\left\langle\rho^{*}(i, j)\right\rangle_{(i, j) \in \mathcal{A}^{c}, i \neq j}$,

where $\mathcal{A}=\{(i, j): i, j \in \mathcal{W}, i \neq j\}$ and $\mathcal{A}^{c}$ denotes its complementary set. Thus, a stable synfire chain is described as $C C_{\text {syn }}$ is large, near 1 and $C_{\text {outside }}$ is small so that $C C_{\text {syn }}-C C_{\text {outside }}$ is distinctly apparent.

It can be seen from Fig. $6 \mathbf{A}$ that if $r \neq 1$, in a network of a large size, both the CCs among the synchrony cluster and outside reach a local minimums. Therefore, a balanced network seems not good to conserve the synchronisation among the cluster but optimal to keep the CCs of the background small values. The size of cluster also influence the stability of the synfire chain. As one can see from Fig. $6 \mathrm{~B}$, both the maps of CCs of intra-cluster and outside with respect to the size of the cluster are sigmoid-like. In comparison to the outside, the steep slope of the curve of intra-cluster CC can approach at a smaller size of cluster than that of the outside $\mathrm{CC}$. The parameter area of $m$, where the intra-cluster $\mathrm{CC}$ is much lager than the outside $\mathrm{CC}$, provides the possibility to realize a stable propagation of synfire chain. However, if $m$ is small, both CCs are small but if $m$ is too large, both CCs keep in high values. That is to say, synchronisation in a cluster of a relative small size may collapse for several layers but synchronisation in a cluster of a large size may diffuse through the whole network. By a mean-field approach (not shown in this paper), we can obtain an approximation

$$
W \sim \sqrt{K}
$$

as the size of the whole network goes to infinity, which is optimal for a stable synfire chain. In addition, the ratio $W / \sqrt{K}$ can neither too small nor too large.

It has been illustrated [12] that increasing the firing rate leads that the map approach the identity map. We use the parameter $b$ in the CC map to show how the firing rate influence the synchronisation. Fig. $6 \mathbf{C}$ illustrates the shapes of the map depending on $b$, which implies that increasing $b$ can make the map approach the identity map too. So, we claim that a larger $b$ implies a higher firing rate in the network. One can see from Fig. $6 \mathbf{D}$ that the ascent of $b$ (increasing firing rates) enhances the CCs of both intracluster and the outside. As a result, a stable synfire chain can occur for a small size of cluster if we increase the firing rate $(b)$ but it will lead diffusion of synchrony at a smaller size of cluster in comparison to the lower firing rates. Instead, a synfire chain may collapse for a smaller size of cluster but can exist for a lager size of cluster, in comparison to the spiking background of higher firing rates. These phenomena can be supported by simulating LIF spiking neuronal network. But the details are omitted in this paper according to the limit of space and will be presented in anywhere else.

In summary, we can conclude:

1) $r \approx 1$, a nearly balanced network counts for preventing the synchronisation diffusing out of the cluster;

2) a suitable size of cluster can prevent the synchronisation from collapse and diffusion;

3) a higher firing rate can enhance synchronisation as well as its diffusion.

\section{COnClusions}

The LIF model network provides a powerful computational tool in both theoretical neuroscience and dealing the experimental data. However, it is still complicated to perform rigorous mathematical analyses. A widely-used simplification is to approximate spike processes as Gaussian processes, keeping the first and the second order moment identical. Thus, the dynamics of the subthreshold membrane potential of a LIF neuron can be described as an OU process. The theory of stochastic dynamical system can be employed. Different from the existing work, which usually only take the membrane potential or the mean firing rate into considerations, we develop a novel model based such an OU approximation (moment neuronal network approach) to describe the dynamics of both the first- and second-order statistics of the spike counting. By this way, a random Gaussian field of neuronal network is established. Also, we present some preliminary analytic results on the dynamics of the model, which provide qualitative analyses of several important phenomena in both biological experimental observes and LIF neuronal network. We find, if the inhibition is stronger than excitation, the firing rate response to the input firing rate is 
not sigmoidal or even not monotonic any more, but bends like a tent map, which could lead fruitful dynamic behaviors. This observation leads an apparent difference between our model and the classic WCA model. Also, our model illustrates the spontaneous activity, which has been widely observed in biological experiments. The description of the dynamics of the correlation coefficient (CC) provides a tool to study another widely-observed phenomenon,synchronisation propagation, in the neuronal network, to which the WCA model is clearly unavailable. By this manner, we illustrate how the three parameters affect the stability of a synfire chain.

We argue that this model can facilitate the analyzing the dynamics in spiking neuronal networks and due to introducing the second-order statistics, a number of statistic tools can be available to tackle with biological data via this model. More important, the framework of this model can be generalized to other spiking neuronal network model, for example, nonlinear LIF neuron model and Hodgkin-Huxley model, via the first-passage-time theory [28]. We also note that the current model has shortcomings which may prevent its applications to quantitatively deal with the real experiment data, despite of its qualitative analysis capability to several experimental phenomena. On the one hand, the LIF model is too simple, without taking other important quantities such as the AMPA and GABA currents into consideration. On the other hand, the MNN maps are already complicated for both analytic and numerical discussions, which demands to further simplify the model without affecting the dynamic properties but decreasing the complexity. Those are our current research topics.

\section{ACKNOWLEDGMENT}

W. L. Lu is supported by the Foundation for the Author of National Excellent Doctoral Dissertation of PR China No. 200921, the National Natural Sciences Foundation of China under Grant No. 60804044, and the Shanghai Pujiang Program No. 08PJ14019, and J. F. Feng is supported by an EPSRC (UK) grant CARMAN. We also acknowledge the financial support of the Future and Emerging Technologies (FET) programme within the Seventh Framework Programme for Research of the European Commission, under the FET-OPEN grant agreement BION, number 213219.

\section{REFERENCES}

[1] P. Dayan and L. Abbott, Theoretical neuroscience: computational and mathematical modelling of neural systems, Boston: MIT Press, 2002.

[2] A. Destexhe and D. Contreras, "Neuronal computations with stochastic network states", Science, vol. 314, pp. 85-90, 2006.

[3] J. F. Feng (editor), Computational Neuroscience-A Comprehensive Approach, FL: Chapman and Hall/CRC Press, London/Boca Raton, 2003.

[4] L. Lapicque, "Recherches quantitatives sur lexcitation electrique des nerfs traitee comme une polarization", J. Physiol. Pathol. Gen., vol. 9, pp. 620-635, 1907.

[5] A., Hodgkin and A. Huxley, "A quantitative description of membrane current and its application to conduction and excitation in nerve", $J$. Physiol., vol. 117, pp. 500-544, 1952.

[6] H. R. Wilson and J. D. Cowan, "Excitatory and inhibitory interactions in localized populations of model neurons", Biophys. J., vol. 12, pp. $1-24,1972$.
[7] H. R. Wilson and J. D. Cowan, "A mathematical theory of the functional dynamics of cortical and thalamic nervous tissue", Kybernetik, vol. 13, pp. $55-80,1973$.

[8] S. Amari, "Homogeneous nets of neuron-like elements", Biol. Cybern., vol. 17, pp. 211-220, 1975.

[9] S. Amari, "Dynamics of pattern formation in lateral-inhibition type neural fields", Biol. Cybern., vol. 27, pp. 77-87, 1977.

[10] W. Erlhagen and G. Schöner, "Dynamic field theory of movement preparation”, Psych. Rev., vol. 109, pp. 545-572, 2002.

[11] J. F. Feng, Y. Deng, and E. Rossoni, "Dynamics of moment neuronal networks", Phys. Rev. E, vol. 73, 041906, 2007.

[12] J. De La Rocha, J. B. Doiron, E. Shea-Brown, K. Josic, and A. Reyes, "Correlation between neural spike trains increases with firing rate", Nature, vol. 448, pp. 802-806, 2007.

[13] G. Deco, V. K. Jirsa, P. A. Robinson, M. Breakspear, K. J. Friston, "The dynamic brain: from spiking neurons to neural masses and cortical fields", PLoS Comp. Biol., vol. 4(8), e1000092, doi:10.1371/journal.pcbi.1000092.

[14] R. Vanmarke, Random Fields: Analysis and Synthesis, Cambridge, Massachusetts: The MIT Press, 1983.

[15] J. F. Feng and D. Brown, "Impact of correlated inputs on the output of the integrate-and-fire models", Neural Computation, vol. 12, pp. 671692, 2000.

[16] R. Romo, A. Hernandez, and A. Zainos, "Neuronal correlates of a perceptual decision in ventral premotor cortex", Neuron, vol. 41, pp. 165-173, 2004.

[17] R. Miles, R. Traub, and R. Wong, "Spread of synchronous firing in longitudinal slices from the CA3 region of hippocampus", J. Neurophysiol, vol. 60, pp. 1481-1496, 1995.

[18] A.D. Reyes, "Synchrony-dependent propagation of firing rate in iteratively constructed networks in vitro", Nat. Neurosci., vol. 6, pp.593, 2003.

[19] D. R. Cox, Renewal theory, Lodon: Methuem \& CO LTD, 1962.

[20] L. Gammaitoni, et.al., "Stochastic resonance", Rev. Mod. Phys., vol. 71, pp. 233-287, 1998.

[21] W. Lu, R. Enrico, and J. F. Feng, "Towards a Theory of Random Neuronal Field", NeuroImage, in press, 2010, doi:10.1016/j.neuroimage.2010.02.075.

[22] P. Fatt and B. Katz, "Spontaneous subthreshold activity at motor nerve endings", J Physiol., vol. 117:1, pp. 109-208, 1952.

[23] M. Tsodyks, T. Kenet, A., Grinvald, and A. Arieli, "Linking Spontaneous Activity of Single Cortical Neurons and the Underlying Functional Architecture", Science, Vol. 286, pp. 1943-1946, 1999.

[24] Y. Ikegaya, et.al., "Synfire Chains and Cortical Songs: Temporal Modules of Cortical Activity", Science, vol. 304, pp. 559-564, 2004.

[25] A. Aertsen, M. Diesmann, M. O. Gewaltig, "Propagation of synchronous spiking activity in feedforward neural networks", J. Physiology, vol. 90, pp. 243-247, 1996.

[26] M.-O. Gewaltig, M. Diesmann, A.Aertsen, "Propogation of cortical synfire activity: survival probability in single trials and stability in mean", Neural Network, vol. 14, pp. 657-673, 2001

[27] Y. Aviel, C. Mehring, M. Abeles, and D. Horn, "On Embedding Synfire Chains in a Balanced Network", Neural Computation, vol. 15, pp. 13211340, 2003.

[28] H. C. Tuckwell, Introduction to Theoretical Neurobiology. Cambridge Univ. Press: UK, Cambridge, 1988. 